\section{Key challenges to the incorporation of climatic design knowledge in contemporary tropical architecture}

Climate is inseparable from the environmental context of the built environment. It determines the nature and severity of the environmental forces that impinge on the building envelope and influences the soil as well as the flora and fauna of the surroundings. Climate also influences the social milieu in which design occurs: the all inclusive concept of "culture" is heavily influenced by climate and is woven around the seasons, extremes and variations in weather parameters. In most cases, the building's brief (i.e. programme) does (or at least ought to) reflect the climatic context in which the building is created. Economy of design can only occur if the built environment works WITH climate and not AGAINST it.

However, there are several challenges in applying climate knowledge to the creation of built environment in a manner conducive to sustainable living, especially in the urban tropics. The design standards and expectations are rapidly evolving in the context of unprecedented urban population growth. Climate itself is changing, more so in the rapidly growing tropical megacities (global as well as meso- and micro-climate changes). Climate data are not readily available, or when available, not readily usable by building designers. The spatial and temporal scales at which climate data are needed by the creators of urban and built environment are not easy (or cheap) to produce. To confound matters further, the interaction between climate and the built environment is governed by the uncertainties of global and regional climate change augmented by the inadvertent climate consequences of urban built environment.

At the same time, a considerable body of knowledge exists with respect to climate-sensitive design contextualised for tropical urban climates. Although the field of inquiry is relatively young, we now know enough to be able to utilise this knowledge to enhance the climatic quality of designed spaces. In this light, it is pertinent to focus on key challenges to the utilization of existing knowledge to contemporary design practices in the urban tropics. Three possible issues are posited as key to the utilization of knowledge already gained. They also point to key research and design challenges for the immediate future. i. Design strategies - especially to pro mote shading while facilitating ventilation

ii. Nexus between climate-sensitive design and urban transportation

\section{ii. Data needs in the urban tropics}

The amelioration of the urban climate anomaly in the tropics increasingly point to the need for shade. For too long the emphasis has been on ventilation, to the detriment of all other climatesensitive design options. While there is no denying of the cooling effect of urban breeze, wind's cooling potential is greatly enhanced if accompanied by shade. Coupled with the difficulties arising out of increasing built density, need for privacy and poor air quality, ventilation strategies by themselves will struggle to achieve the desired comfort. It is necessary to link them to other design approaches, especially shading.

A second challenge is to consider the links to transportation requirements. All urban activities start and end on foot. And in high density cities what goes in between is more likely to be on foot than on any other mode. Yet the provision of transportation remains divorced from the rest of the endeavours to create climatically sensitive and quality urban spaces. We need to move in the direction of high-density development and pedestrianization simultaneously. Higher density living in turn has consequences for shading and ventilation patterns and this needs imaginative design and planning solutions.

A third challenge is to develop adequate and relevant urban climate data. A critical need of the built environment professional is reliable and detailed local (and meso scale) climate data especially in urban areas. It is also necessary to provide detailed climatic information as databases in Geographic Information System (GIS) platforms and in the form of digital elevation models (DEMs). Reliable and detailed stand-alone data files usable as input files for building simulation are also needed.

R Emmanuel Editor 